\title{
A robust electrophysiological marker of spontaneous numerical
}

\section{discrimination}

\author{
Carrie Georges $^{\mathrm{a}^{*}}$, Mathieu Guillaume ${ }^{\mathrm{b}}, \&$ Christine Schiltz ${ }^{\mathrm{a}}$
}

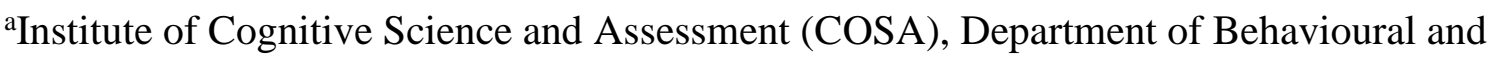
Cognitive Sciences (DBCS), Faculty of Humanities, Education and Social Sciences (FHSE),

University of Luxembourg, Campus Belval, Maison des Sciences Humaines, Porte des

Sciences 11, L-4366 Esch-sur-Alzette, Luxembourg.

${ }^{\mathrm{b}}$ Center for Research in Cognitive Neuroscience (CRCN), Université Libre de Bruxelles, Avenue Franklin Roosevelt 50 (CP 191), 1050 Brussels, Belgium.

C.G. and M.G. have contributed equally to this work and share first authorship.

(1)

*Correspondence should be addressed to: 


\section{Abstract}

24 Humans have a Number Sense that enables them to represent and manipulate numerical

25 quantities. Behavioral data suggest that the acuity of numerical discrimination is predictively

26 associated with math ability - especially in children - but some authors argued that its

27 assessment is problematic. In the present study, we used frequency-tagged

28 electroencephalography to objectively measure spontaneous numerical discrimination during

29 passive viewing of dot or picture arrays in healthy adults. During one-minute sequences, we

30 introduced periodic numerosity changes and we progressively increased the magnitude of

31 such changes every ten seconds. We found significant brain synchronization to the periodic

32 numerosity changes from the 1.2 ratio over medial occipital regions, and amplitude strength

33 increased with the numerical ratio. Brain responses were reliable across both stimulus

34 formats. Interestingly, electrophysiological responses also mirrored performances on a

35 number comparison task and seemed to be linked to math fluency. In sum, we present a

36 neural marker of numerical acuity that is passively evaluated in short sequences, independent

37 of stimulus format and that reflects behavioural performances on explicit number comparison 38 tasks.

39 Number word count: 170. 
We can effortlessly approximate how many people are in a given room or how many

objects form a collection. Dehaene postulated that humans have a Number Sense, a cognitive ability that allows representing and manipulating large numerosities (Dehaene, 2001). This intuitive understanding of numbers has been defined as a cognitive system dedicated to number processing, the Approximate Number System (ANS, Feigenson, Dehaene, \& Spelke, 2004). The acuity of this cognitive system is characterized by scalar variability (Gallistel \& Gelman, 2000) so that mental representations of large numerosities are less precise than those of small numbers. It is assumed that this system follows the Weber-Fechner law (Dehaene, 2003; but see Cantlon, Cordes, Libertus, \& Brannon, 2009; and Piantadosi, 2016; for alternative views). Accordingly, comparing two quantities depends on the numerical ratio between them (e.g., 10 and 20 objects are as distinguishable as 20 and 40 objects). In the literature, ANS acuity is commonly assessed through non-symbolic number comparison tasks, with the underlying idea that better numerical discrimination results from more precise number representations, that is, greater ANS acuity. Much attention has been devoted to this cognitive system since the observation that ANS acuity predicted adolescents' arithmetic performance throughout their scholarship (Halberda, Mazzocco, \& Feigenson, 2008). Some studies also reported a close relationship between ANS acuity and mathematical ability (in children, $r=.54$ in Inglis, Attridge, Batchelor, \& Gilmore, 2011; $r=.35$ in Mussolin, Mejias, \& Noël, 2010; $r=.52$ in adults,

60 DeWind \& Brannon, 2012), but other studies did not report such a correlation (e.g., 61 Sasanguie, Göbel, Moll, Smets, \& Reynvoet, 2013; Price, Palmer, Battista, \& Ansari, 2012; see Chen \& Li, 2014; and see Schneider, Beeres, Coban, et al., 2016; for meta-analyses). This

63 discrepancy has been attributed to ambiguities relative to the number comparison task

64 (DeWind \& Brannon, 2016; Norris \& Castronovo, 2016; Szücs, Nobes, Devine, Gabriel, \& 
Gebuis, 2013). In such a task, participants strategically use all available information from the

66

67

68

69

visual scene to make their decision (Roquet \& Lemaire, 2019; see also Gebuis, Cohen

Kadosh, \& Gevers, 2016; and Leibovich, Katzin, Harel, et al., 2016 for extensive discussions about the interplay between numerical and non-numerical processes). This has led some authors to suggest that comparison tasks mostly involve executive processes (notably inhibition) at the expanse of numerical processes (Cragg \& Gilmore, 2014; Gilmore et al., 2013). The non-negligible involvement of executive functions in number comparison tasks could thus disqualify their validity of indexing basic numerical processes (Smets, Gebuis, Defever, \& Reynvoet, 2014; see also Guillaume et al., 2020). To adequately measure ANS acuity, one therefore needs the possibility to assess numerical discrimination without any explicit task to thereby prevent deliberate judgements based on non-numerical information. Recent studies found that humans can spontaneously perceive number from a visual scene, without any explicit task related to number processing (Anobile, Guerrini, Burr, et al., 2019; Cicchini, Anobile, \& Burr, 2016). These observations support the idea that numerosity is a salient property of the visual environment. It was even further proposed that number is a topologically invariant property, independent from other visual percepts (Kluth \& Zetzsche, 2016). This view is notably supported by deep-network modeling showing that numerosity emerges as a statistical property of pictures in hierarchical generative models (Stoianov \& Zorzi, 2012). Burr and Ross already emphasized in 2008 that numerosity is a primary visual property, easily captured by a Visual Number Sense (Burr \& Ross, 2008). The ability to extract numerical information from a visual scene is now considered as a universal ability shared with other animal species (Ferrigno, Jara-Ettinger, Piantadosi, et al., 2017). Recent neuropsychological data provide evidence that specific visual mechanisms capture numerosity independently of cognitive control (Anobile, Tomaiuolo, Campana, et al., 2020). 
Such visual processes dedicated to numerical extraction could thus be an interesting proxy of ANS acuity and more generally numerical ability.

In the current study, we intended to measure the ability to spontaneously extract numerical information from visual scenes by recording brain responses to numerical fluctuations during a relatively short passive viewing task that did not involve any explicit numerical processes. Furthermore, we aimed at comparing brain responses to such numerical information in the context of either simple geometrical forms (i.e., dot arrays) or richer colourful pictures. To achieve these goals, we adapted a paradigm based on the Fast Periodic Visual Stimulation (FPVS) method (Rossion, 2014).

The FPVS approach is based on the observation that the human brain synchronizes its activity to the periodic state of a flickering stimulus (Adrian \& Matthews, 1934), leading to Steady-State Visual Evoked Potentials (Regan, 1977). By introducing a periodic fluctuation of a target stimulus feature during a sequence of passively viewed stimuli, we can favourably use this synchronization property of the brain to record cerebral responses specific to the periodic feature manipulation. Indeed, FPVS provides an objective measure of brain sensitivity to the periodic changes of identity (Norcia, Appelbaum, Ales, et al., 2015; Regan, 1989). Many studies used the FPVS design to investigate neural discrimination of face identities (Liu-Shuang, Norcia, \& Rossion, 2014; Retter \& Rossion, 2016), facial expressions (Dzhelyova, Jacques, \& Rossion, 2016), letters and words (Lochy, Van Belle, \& Rossion, 2015; Lochy, Jacques, Maillard, et al., 2018), tool categories (De Keyser, Mouraux, Quek, et al., 2018), and digits (Guillaume, Poncin, Schiltz, et al., submitted). We recently found (Guillaume, Mejias, Rossion, et al., 2018; Van Rinsveld, Guillaume, Kohler, et al., 2020) that FPVS can also provide a reliable electrophysiological measure of numerical discrimination independent from other visual properties (see also Park, 2017; Lucero, Brookshire, Sava- 
113 Segal, et al., 2020; for a similar observation). These findings make FPVS a valuable tool to

114 measure numerical discrimination without requiring any explicit task.

115 In the current study, we recorded electrophysiological responses to periodic changes

116 of numerosity in a fast stream of visual stimuli following a sinusoidal contrast modulation at

$11710 \mathrm{~Hz}$ (see Figure 1A). We manipulated the number of displayed items so that it

118 systematically switched from ten to another number. The carrier (i.e., ten) alternated with the

119 second number at the fluctuating rate of $5 \mathrm{~Hz}$. The value of the second number progressively

120 increased during the one-minute sequence, from ten to fifteen. The numerical increment

121 occurred every ten seconds. The numerical ratio between the carrier and the second number

122 thus raised from 1.0 to 1.5 (with a step of 0.1 ) across six continuous ten-second periods. This

123 method using a progressive numerical increment is a significant improvement of our previous

124 method where we assessed brain responses to numerical ratios in different conditions

125 (Guillaume et al., 2018; Van Rinsveld et al., 2020). Here the progressive numerical increment

126 allowed us to determine in one sequence the first ratio at which numerical discrimination was

127 achieved at the brain level. In this respect, electrophysiological responses tagged at $5 \mathrm{~Hz}$ (i.e.,

128 the frequency of the alternating change) are an individual and objective neural marker of 129 numerical discrimination.

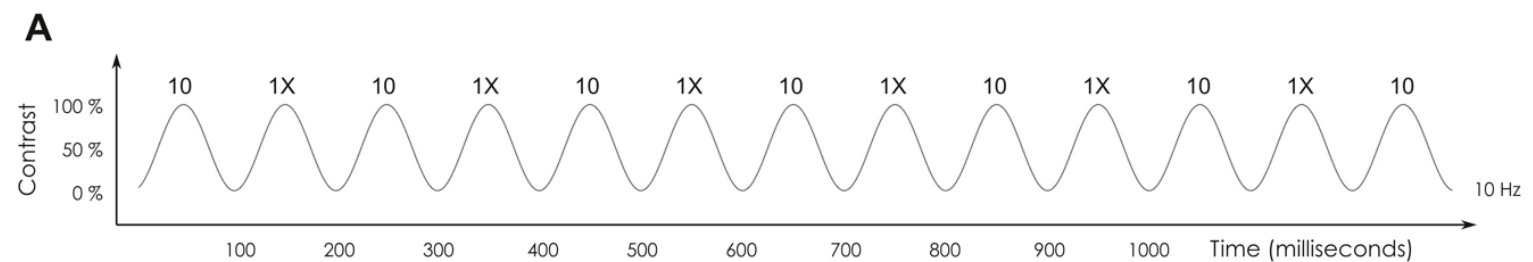

B Dots
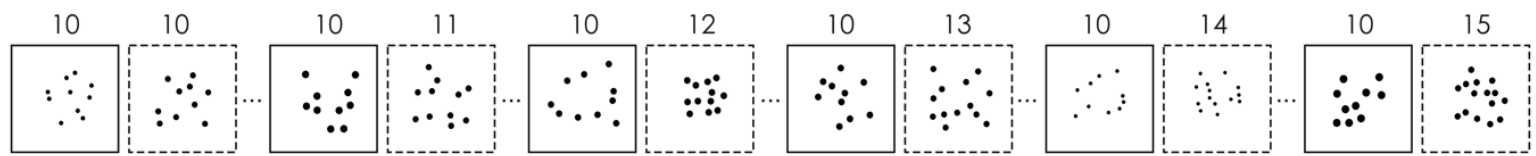

Pictures

130
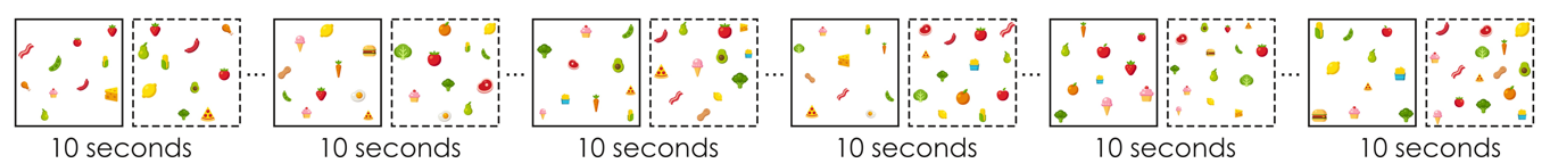
131 Figure 1. Illustration of the experimental design. (A) The onset and the offset of the stimuli followed a sinusoidal contrast stimulation (from blank to full luminance) at $10 \mathrm{~Hz}$. There was a $5 \mathrm{~Hz}$ alternation between the carrier (10) and a second number (1X). (B) In two different conditions (cautiously designed dots or colourful pictures), the value of the second number increased every ten seconds, from 10 to 15 .

We designed two stimuli sets for the FPVS sequences: a first set of simple but carefully constructed items and a second set intended to be more complex but also more appealing (see Figure 1B for illustrations). The first set consisted in dark dot arrays with homogeneous dot sizes. For this set, we meticulously controlled, at the sequence level, for unwanted periodic changes related to several potentially interfering visual features (as in Van Rinsveld et al., 2020). Conversely, the second set was intended to be richer and as such composed of colourful food drawings of heterogeneous sizes. We deliberately did not control for any visual features in this condition. On the contrary, it included even more complex visual changes in that quite a few items contained varying numbers of sub-items (e.g., there are three pepperonis on the pizza picture). Comparing these two sets allowed us to verify whether the neural marker of numerical discrimination is robust to stimulus complexity, thus providing us with a reliable measure of spontaneous numerical information processing.

\section{Results}

\section{Numerical acuity and Math fluency}

In the non-symbolic number comparison task, participants were able to determine the larger of the two dot arrays with a mean accuracy of $91.07 \%$ (Standard Deviation $(S D)=$ $2.93 \%)$, and they yielded the correct response on average in $570 \mathrm{~ms}(S D=77 \mathrm{~ms})$.

Accuracies ranged from $77.53 \%$ to $98.51 \%$ and correct RTs from $647 \mathrm{~ms}$ to $524 \mathrm{~ms}$ for the closest (i.e., 1.1) and the most distant (i.e., 1.6) ratios respectively (see Figure 3C). The mean value of the Weber fraction was $.116(S D=.03)$. In the math fluency task, average performance across all five subtests was $75 \%(S D=13.55 \%)$, with an averaged raw score of 150 out of 200 . 


\section{Instruction compliance}

We assessed compliance by instructing participants to keep their gaze on a fixation

160 diamond displayed at the centre of the screen. The diamond randomly (between six to eight

161 times) changed color during the sequences, and participants were instructed to press a button

162 upon detecting the change (as in Guillaume et al., 2018, see Methods). Participants overall detected the color change in $509 \mathrm{~ms}(S D=50 \mathrm{~ms})$ with only $4.24 \%(S D=3.41 \%)$ of misses across both the dots and pictures conditions. Such a relatively high detection rate $(>95 \%)$ indicates that participants kept their gaze on the centre of the screen during the EEG acquisition.

\section{Topographies and Signal-to-noise ratio}

Figure 2 depicts the topographies of the cerebral responses specific to the numerical discrimination of the alternating change. Accordingly, we computed the Baseline-Corrected Amplitudes (BCA, see Methods) at the frequency of interest (i.e., $5 \mathrm{~Hz}$ ) for every ratio (i.e.,

171 for each 10-second segment) in each condition (Figure 2A: Dots condition; Figure 2B:

172 Pictures condition). We separately computed BCAs for every participant and then averaged the amplitudes at the inter-individual level. No responses at $5 \mathrm{~Hz}$ were observed at the scalp level for a ratio of 1 (i.e., no change) in any of the two conditions. Conversely, for numerical ratios equal to and greater than 1.2, strong peaks were recorded in posterior regions, mostly

176 in the medial occipital area centered around the electrodes $\mathrm{Iz}, \mathrm{O} 1, \mathrm{O} 2$ and $\mathrm{Oz}$ (see

177 Supplementary Table 1 for comparably lower frequency-tagged EEG responses in the left, medial, and right occipito-parietal cortices). Since similar response patterns were observed on each of these medial occipital electrodes (see Supplementary Figure 1), further analyses were

180 limited to the electrode $\mathrm{Oz}$ for reasons of brevity and to be in accordance with previous 
studies consistently reporting large number-specific effects on that electrode (see e.g.,

182 Guillaume et al., 2018; Lucero et al., 2020; Park, 2017; Van Rinsveld et al., 2020).

184 EEG spectra recorded on the medial occipital electrode $(\mathrm{Oz})$ for every ratio in the dots and

185 pictures conditions. SNR peaked at $10 \mathrm{~Hz}$ for every ratio in each condition (across all ratios:

186 dots: mean $=7.46, S D=0.38$; pictures: mean $=7.68, S D=0.32$ ). This is expected since these

187 cerebral responses reflect the visual contrast between the background and the stimuli induced

188 by their periodic onset at $10 \mathrm{~Hz}$. Most importantly, there was a very clear brain response at 5

$189 \mathrm{~Hz}$ on $\mathrm{Oz}$ associated with a numerical ratio equal to and greater than 1.2 for both dots and

190 pictures. The SNR seemed to progressively increase with increasing numerical ratio in both

191 conditions.

\section{A DOTS}
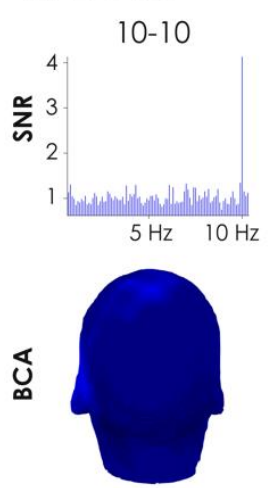

B PICTURES
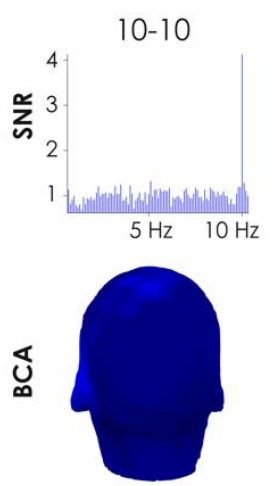
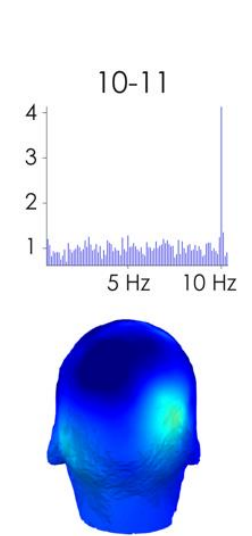

10-11
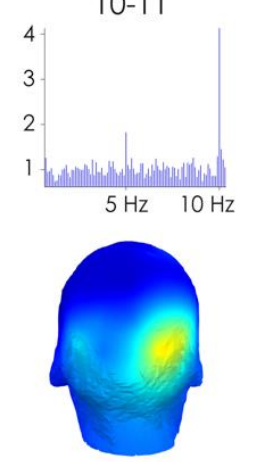
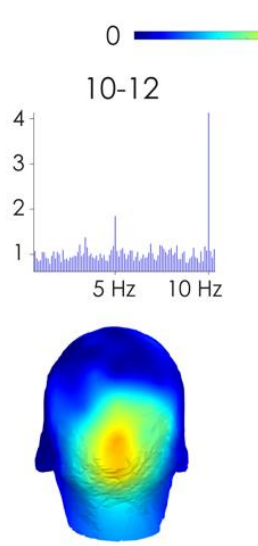$$
0
$$

10-12
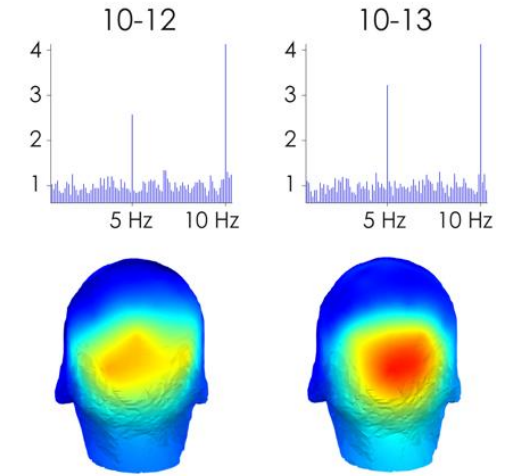
$0.25 \mu \mathrm{V}$
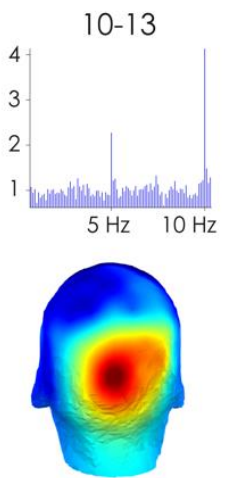
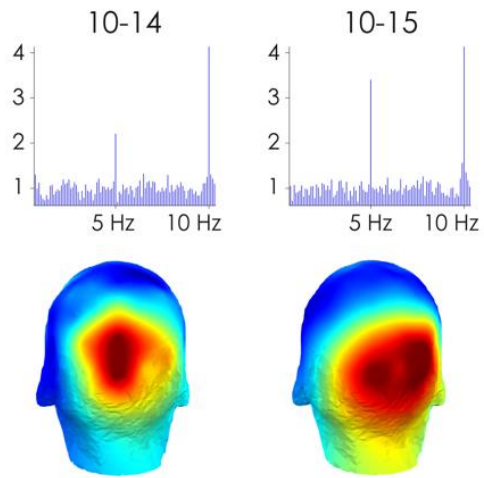

192

Figure 2. Brain responses to numerical changes for $(A)$ the Dots condition and $(B)$ the Pictures condition. 
To assess the statistical significance of the cerebral responses synchronised to

199 numerical changes, we computed a Z-score of the response at $5 \mathrm{~Hz}$ for every ratio in each

200 condition per participant (see Methods). A Z-score value larger than the threshold of 1.64 ( $p$

$201<.05$, one-tailed, testing signal level > noise level) indicates a significant cerebral response to

202 the number change, and thus measures successful numerical discrimination at the neuronal

203 level.

204 We found that the EEG signal tagged at $5 \mathrm{~Hz}$ on the medial occipital electrode $\mathrm{Oz}$

205 averaged across all participants was significantly above the noise level from a numerical ratio

206 of 1.2 onwards in both the dots and pictures conditions (see Figure 3A \& 3B). This suggests

207 that visual number discrimination was successfully achieved from the ratio 1.2. Note that this

208 finding is consistent with our behavioural data yielding an averaged Weber fraction value of

209 .116. This value indicates accurate (i.e., more than $75 \%$ correct) number discrimination from

210 a ratio of 1.116 onwards, corresponding to significant EEG responses at the 1.2 ratio, which

211 was the next ratio sampled after 1.1 in our EEG design. In other words, our FPVS results are

212 comparable to the behavioural performances on the number comparison task, indicating

213 accuracies significantly greater than $75 \%$ from a ratio of 1.2 onwards (see Figure 3C).

214 Further, EEG signal amplitudes seemed to progressively increase with increasing numerical

215 ratio in both conditions. 

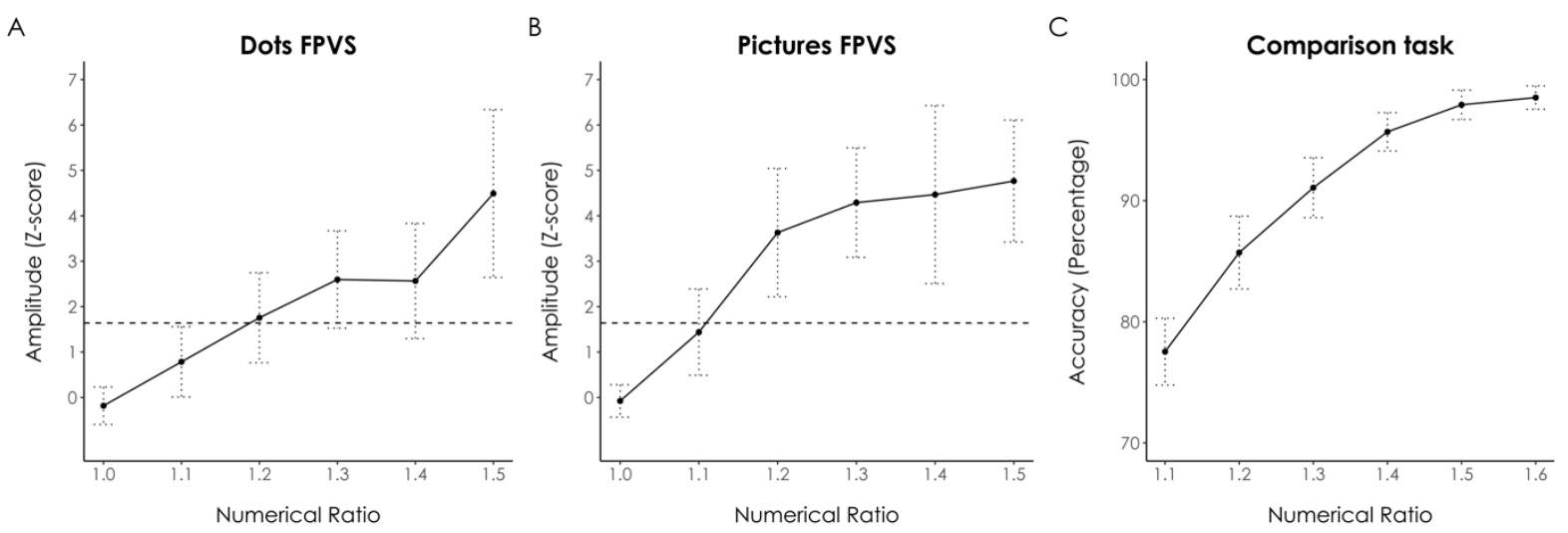

Figure 3. EEG and behavioural data depiction. Amplitudes of $\mathrm{Oz}$ (in Z-score) in (A) the Dots condition and

(B) the Pictures condition as a function of the numerical ratio. The horizontal line depicts the $95 \%$ significant threshold (i.e., 1.64) above the noise level. (C) Behavioural data from the non-symbolic number comparison task (accuracy, in percent). Vertical lines depict 95\% Confidence Intervals in each graph.

Overall, the tagged cerebral response on the medial occipital electrode $\mathrm{Oz}$ was stronger for pictures $(\mathrm{Z}$-score $=3.09, S D=3.32)$ than dots $(\mathrm{Z}$-score $=2.00, S D=2.89)$. We conducted linear mixed effect model analyses to determine whether the brain responses depended on the condition and/or the numerical ratio. We entered the condition and the ratio as fixed effects, with an interaction term, and we included intercepts for participants as random effect. Model comparisons between the full model and reduced models were done using chi-squares tests on the log-likelihood values. Since the full model did not provide a better fit than the reduced model without the interaction term, $\chi 2(1)=0.60, p=.44$, we proceeded with contrasting the model without interaction term to models that were further reduced by excluding the remaining effects in question. Model fits were significantly worse when excluding either condition, $\chi 2(1)=14.82, \mathrm{p}<.001$, or ratio, $\chi 2(1)=99.91, \mathrm{p}<.001$, which indicates that both factors (i.e., condition and the numerical ratio) had significant effects on the brain response.

To further assess the effect of numerical ratio on the tagged cerebral responses, and to verify whether the apparent increase in amplitudes across the ratios was significant, we conducted linear regression analyses to predict the EEG signal at $5 \mathrm{~Hz}$ on $\mathrm{Oz}$ as a function of 
numerical ratio. Results indicated significant positive trends for both dots (adjusted $r^{2}=.93, \beta$

$238=8.44, \mathrm{p}<.001)$ and pictures (adjusted $\mathrm{r}^{2}=.82, \beta=9.7, \mathrm{p}=.008$ ). The cerebral response

239 reflecting numerical discrimination thus progressively increased with increasing numerical

240 ratio in both conditions (see Figure 3A \& 3B).

$241 \quad$ Reliability of the response

242 To confirm the robustness of the aforementioned group findings at the individual

243 level and thereby the sensitivity of the current approach, we assessed both inter- and intra-

244 individual reliabilities of the EEG responses at $5 \mathrm{~Hz}$.

245 In terms of inter-individual reliabilities, $48 \%$ and $71 \%$ of the participants featured a

246 significant frequency-tagged EEG signal (i.e., Z-score > 1.64) on Oz at the numerical ratio

2471.2 (i.e., the smallest ratio at which on average significant cerebral responses to numerical

248 discrimination were recorded on $\mathrm{Oz}$ at the group level) in the dots and pictures condition,

249 respectively. This agrees with Guillaume et al. (2018), reporting number-tagged effects in

250 brain amplitudes also in the majority of their participants and clearly suggests that the present

251 group-level cerebral response at $5 \mathrm{~Hz}$ on $\mathrm{Oz}$ at the numerical ratio 1.2 was not driven by a

252 few outlier participants. The proportion of participants featuring significant frequency-tagged

253 EEG signals on $\mathrm{Oz}$ also gradually increased with increasing numerical ratio (see Figure 4).

254 This is to be expected if one assumes that numerical discrimination, as reflected by

255 significant EEG responses at $5 \mathrm{~Hz}$ on $\mathrm{Oz}$, becomes progressively easier with increasing

256 numerical ratio. 


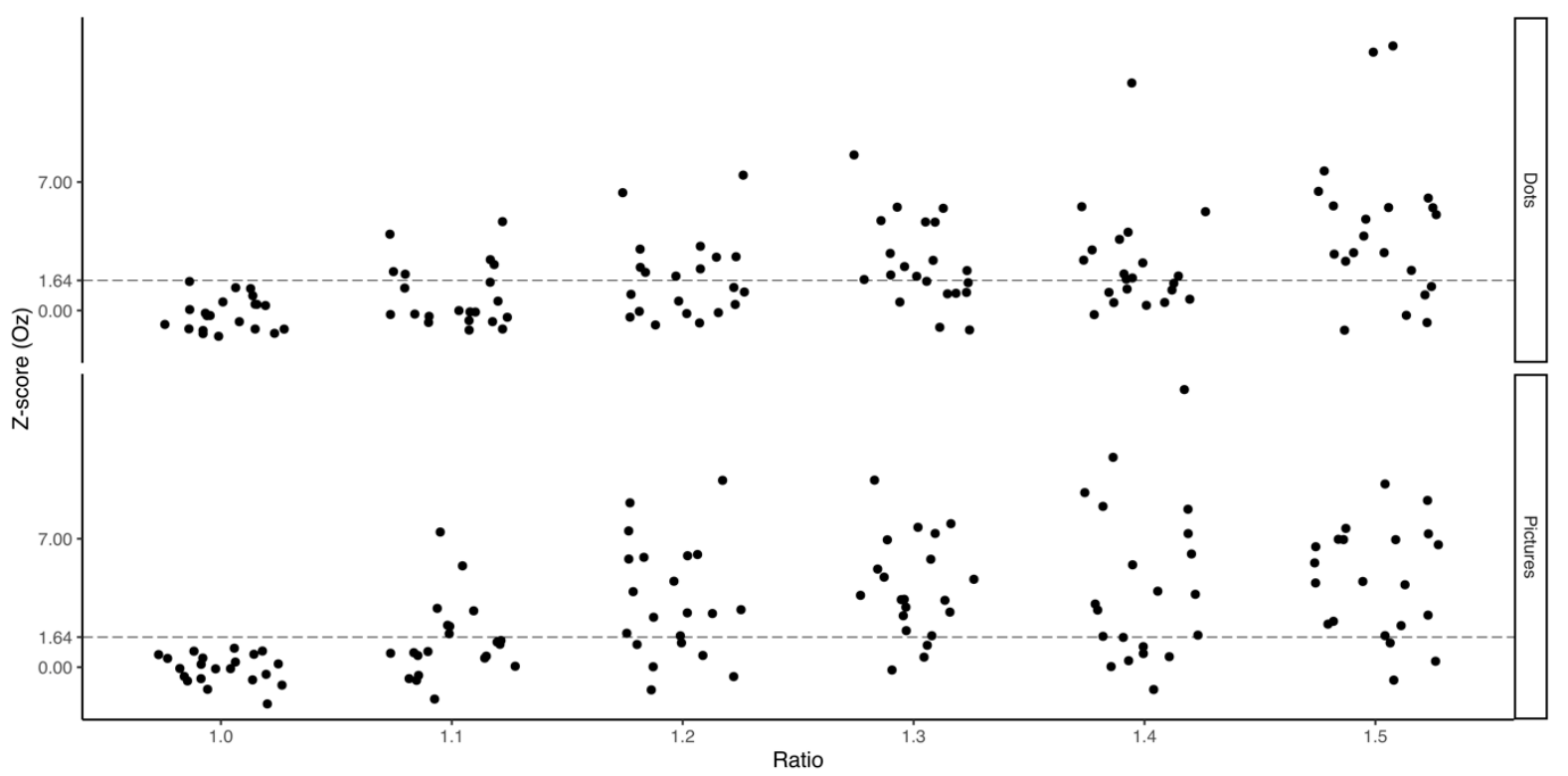

Figure 4. Individual amplitudes of $\mathrm{Oz}$ (in Z-score) as a function of the condition (Dots or Pictures) and the numerical ratio. The vertical dashed line represents the 1.64 threshold of statistical significance at $95 \%$.

It should be noted that when considering for each individual the medial occipital electrode (i.e., Iz, O1, O2, or Oz) at which the largest frequency-tagged response was observed at the ratio 1.2 (instead of focussing on $\mathrm{Oz}$ in every individual), significant numerical discrimination was observed in $57 \%$ and $90 \%$ of the participants at that numerical ratio in the dots and pictures conditions, respectively. When considering the entire posterior scalp, significant cerebral responses at $5 \mathrm{~Hz}$ for the numerical ratio 1.2 were observed in $67 \%$ and $100 \%$ of participants with dots and pictures stimuli, respectively. These data generally confirm the robustness of the present group-level findings at the inter-individual level. cerebral responses on $\mathrm{Oz}$ at the numerical ratio 1.2, considering that it was the lowest ratio

270 yielding significant frequency-tagged EEG signals at the group-level. Intra-individual

271 reliabilities were computed using the odd-even split-half method based on 10 repetitions per condition (see also methods). More concretely, two EEG amplitudes (in Z-score) were computed at $5 \mathrm{~Hz}$ for every individual in each condition and subjected to correlation analysis.

274 The correlation coefficients were Spearman-Brown corrected to get a reliability estimate for 
the entire set of items (i.e., 10 repetitions). Spearman-Brown corrected correlation coefficients were $\mathrm{r}=.33, \mathrm{p}=.41$ and $\mathrm{r}=.68, \mathrm{p}=.02$ in the dots and pictures condition, respectively. When excluding one influential data point with a Cook's distance greater than the conventional cut-off value of 1.0 (i.e., with Cook's distance $=2.1$ ), intra-individual reliability in the dots condition improved to $r=.66, p=.03$. This thus suggests that withinsubject reliabilities of the EEG responses reflecting numerical discrimination were within the acceptable range in both the dots and pictures conditions.

\section{Relationship between the FPVS response and the behavioural measures}

Although this was not one of the main objectives of the present study, we conducted correlation analyses to test whether the EEG signal reflecting numerical discrimination was related to a behavioural measure of numerical discrimination as well as math fluency. Stronger cerebral responses at $5 \mathrm{~Hz}$ on the medial occipital electrode $\mathrm{Oz}$ at the ratio 1.2 in both the dots and pictures conditions were associated with significantly better math fluency (dots: $r=.53, p=.014$; pictures: $r=.51, p=.017$; see Figure 5D and 5E). Conversely, only in the dots condition, a relation was observed between higher EEG signals at $5 \mathrm{~Hz}$ on $\mathrm{Oz}$ at the ratio 1.2 and lower Weber fractions (dots: $r=-.44, p=.047$; pictures: $r=-.20, p=.39$, see Figure 5A and 5B). Notably, the brain responses in the dots condition only marginally correlated with those in the pictures condition $(r=.39, p=.08$; see Figure 5C), suggesting common but also distinct brain processes related to the stimulus complexity. It should also be noted that the values of the Weber fraction did not correlate with math fluency scores in the present study $(r=-.12, p=.62$; see Figure $5 \mathrm{~F})$. When correcting the aforementioned correlations for multiple comparisons using the Holm-Bonferroni step-down procedure, only relations between stronger EEG signals at $5 \mathrm{~Hz}$ on $\mathrm{Oz}$ at the ratio 1.2 in both the dots and pictures conditions and better math fluency remained significant, when considered one-tailed (dots: $p=.042$; pictures: $p=.043$ ). 
301 can be found in the supplementary material.

302
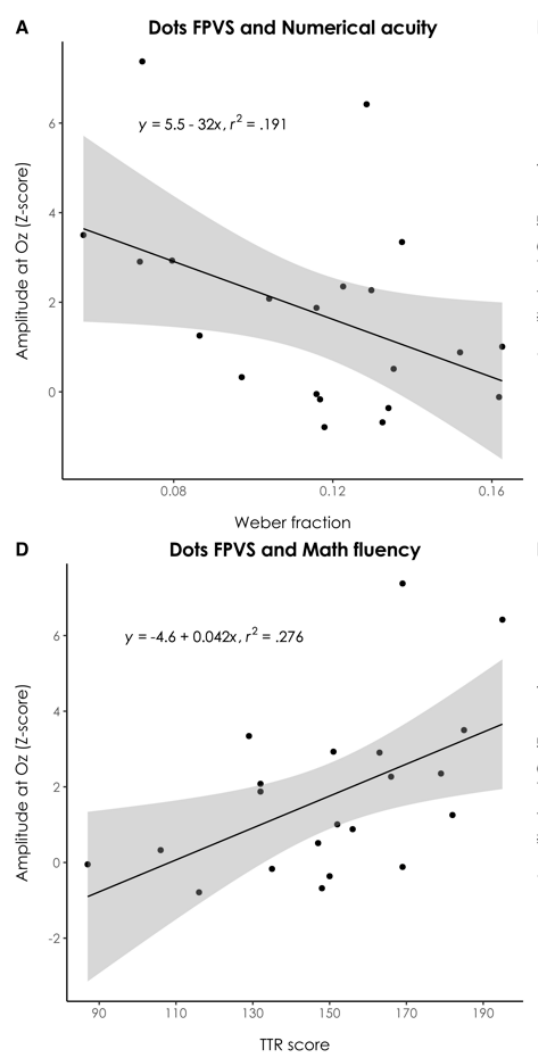
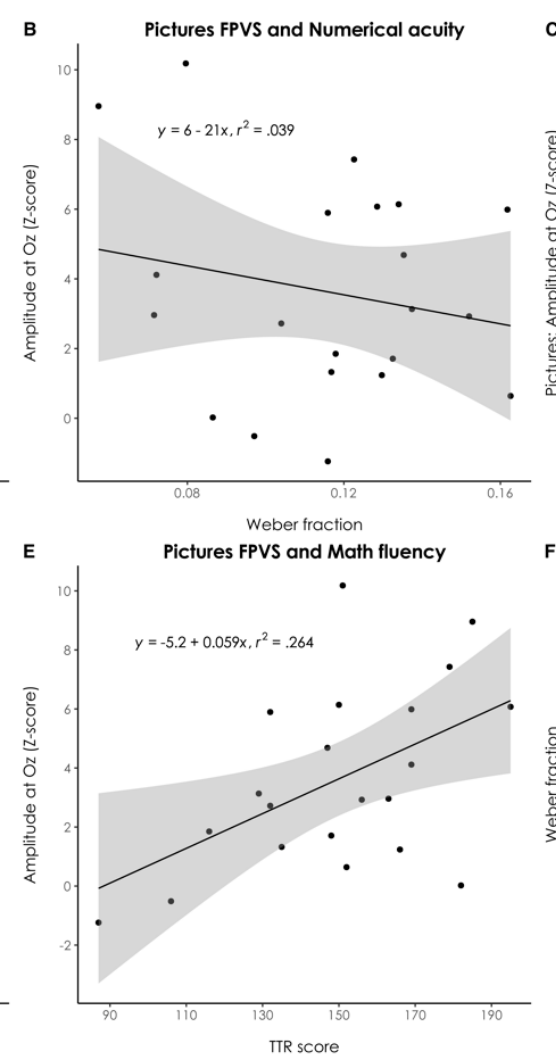

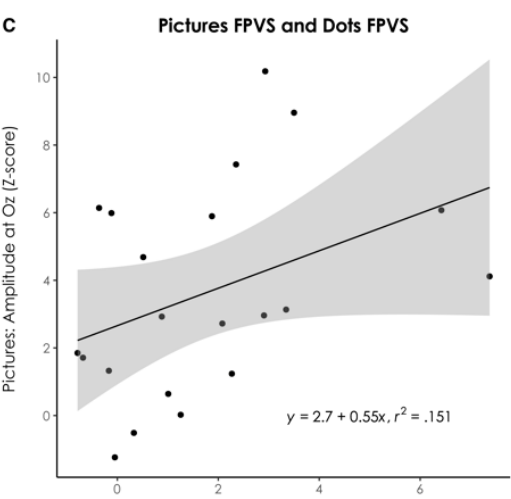

Dots: Amplitude at Oz (in Z-score) Math fluency and Numerical acuity

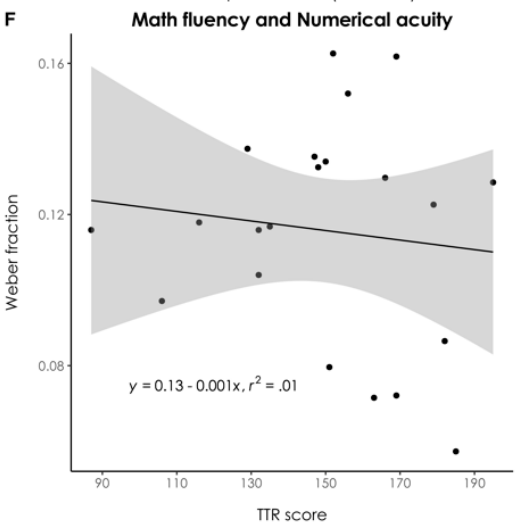

Figure 5. Correlations between EEG and behavioural data. Scatter plots of (A) the values of the Weber

fraction and the amplitudes recorded on $\mathrm{Oz}$ for the numerical ratio 1.2 in the Dots condition and (B) the Pictures condition, (C) the amplitudes recorded on $\mathrm{Oz}$ for the numerical ratio 1.2 in the Dots and Pictures conditions, (D) the math fluency score and the amplitudes recorded on $\mathrm{Oz}$ for the numerical ratio 1.2 in the Dots condition and (E) the Pictures condition, (F) and the values of the Weber fraction and the math fluency scores. The equation of the linear regression line is indicated in each plot. Gray areas depict standard errors.

However, considering the small sample size of the current study for correlation analyses, we acknowledge that the present brain-behaviour relations should be considered with caution without drawing strong conclusions from their outcomes. We therefore complemented the correlation analyses with group comparisons. Since one of the main objectives of the current study was to determine the earliest ratio for which numerical discrimination was achieved at the brain level using both dots and pictures stimuli, we 
315 proceeded by distinguishing individuals based on whether they already featured significant cerebral responses (i.e., Z-score > 1.64) at $5 \mathrm{~Hz}$ on $\mathrm{Oz}$ at the early ratio 1.2 . We conveniently

317 chose this ratio because $48 \%$ of the participants showed significant discrimination

318 (henceforth referred to as "significant signal at 1.2"), while 52\% did not (henceforth referred 319 to as "non-significant signal at 1.2") in the dots condition. We used this balanced grouping

320 index as between-subject factor in an independent t-test analysis. Individuals with significant EEG responses at 1.2 featured significantly better performances in the number comparison task both in terms of overall accuracy (significant signal at 1.2: $92.5 \%$ vs. non-significant signal at 1.2: $89.77 \% ; \mathrm{t}(19)=-2.36, \mathrm{p}=.015$, Cohen's $\mathrm{d}=-1.08$, one-tailed) and of the Weber fraction (significant signal at $1.2: \mathrm{w}=.102 \mathrm{vs}$. non-significant signal at $1.2 \mathrm{:} \mathrm{w}=.128$; $\mathrm{t}(19)=2.26, \mathrm{p}=.018$, Cohen's $\mathrm{d}=1.04$, one-tailed). This group of individuals also showed significantly better math fluency (significant signal at 1.2: sum of correct responses $=160 \mathrm{vs}$. non-significant signal at 1.2: sum of correct responses $=141 ; \mathrm{t}(19)=-1.71, \mathrm{p}=.05$, Cohen's $d=-0.78$, one-tailed). Here, it might also be worth noting that when performing a mediansplit on math fluency scores, only individuals with higher math fluency (i.e., scores above the median value of 151) showed on average significant EEG responses at $5 \mathrm{~Hz}$ on $\mathrm{Oz}$ at the numerical ratio $1.2(\mathrm{Z}$-score $=2.79$ compared to 0.61 in individuals with math fluency scores below the median value of 151). These findings thus collectively replicate the aforementioned correlation outcomes.

We were not able to perform such group comparisons for the pictures condition because here $71 \%$ of the participants showed a significant EEG signal at $5 \mathrm{~Hz}$ on $\mathrm{Oz}$ at 1.2 , and similar grouping would be unbalanced. In any case, in the pictures condition, cerebral responses reflecting numerical discrimination at 1.2 were significantly stronger in those individuals that already showed a significant EEG signal at $5 \mathrm{~Hz}$ on $\mathrm{Oz}$ at the ratio 1.2 in the 
dots condition (significant signal at 1.2: $5.27 \mu \mathrm{V}$ vs. insignificant signal at $1.2: 2.14 \mu \mathrm{V} ; \mathrm{t}(19)$

$340=-2.63, \mathrm{p}=.009$, Cohen's $\mathrm{d}=-1.21$, one-tailed).

\section{Discussion}

In the present study, we were able to extract a cerebral measure of numerical

343 discrimination using an implicit task that only involved passive viewing of numerical

344 quantities, without requiring any explicit numerical judgements. At the group level, participants significantly discriminated between non-symbolic numerosities from the ratio of 1.2 onward. Interestingly, this ratio corresponded to the averaged behavioural performance on the number comparison task, where accuracies were significantly greater than $75 \%$ correct from 1.2 onward. The current study thus successfully yielded an objective neural marker of

349 ANS acuity that directly reflects explicit numerical discrimination in behavioural tasks. Since our marker was extracted during very short sequences, the present study also significantly extents previous observations (e.g., Guillaume et al., 2018) by considerably reducing the amount of time required to determine the sensitivity to numerical discrimination.

Topographical maps revealed that the strongest responses to the periodic numerical changes (at $5 \mathrm{~Hz}$ ) were located in medial occipital regions, with response patterns being very similar between the medial occipital electrodes Iz, O1, O2, and Oz. This is in accordance with previous studies, indicating that numerical information is already extracted at lower levels by visual processes (Anobile et al., 2019; Burr \& Ross, 2008; Cicchini et al., 2016; Park, 2017; Van Rinsveld et al., 2020).

Crucially, the magnitudes of the medial occipital responses linearly increased with the magnitudes of the deviant numerosity (nicely reflecting the architecture of the ANS,

361 Dehaene, 2003). Since we progressively increased the deviant magnitudes during the FPVS 362 sequence, we were able to track the evolution of the brain responses to the periodic number 363 changes at the individual level. We then used the amplitudes of the responses recorded on Oz 
to get an objective neural marker of spontaneous numerical discrimination. Such a marker is desirable because traditional comparison tasks are influenced by non-numerical aspects and involve unwanted deliberate executive processing (e.g., Gebuis et al., 2016; Gilmore et al., 2013; Roquet \& Lemaire, 2019). At the group level, we found that the first ratio where numerical discrimination was achieved at the brain level was 1.2 in the present sample. At that ratio, almost half of the participants (48\%) showed significant numerical discrimination in the dots condition, and a little less than three quarters (71\%) significantly discriminated numerical quantities in the pictures condition, with EEG response amplitudes being relatively reliable at the intra-individual level regardless of stimulus format. It should be noted here that previous works highlighted comparable responses only from a ratio of 1.4 onwards (see

374 Guillaume et al., 2018; Van Rinsveld et al. 2020). This slight discrepancy might be explained by the present participants' high ability to discriminate between numerosities, which was reflected by the comparably low Weber fraction of .116 in the number comparison task (this value is on average .22 in typical adult samples, Guillaume \& Van Rinsveld, 2018). Such an elevated sensitivity to numerical discrimination at the behavioural level in the present sample might then account for the observation that the cerebral responses capturing the numerical change already reached significance at the group level at the early ratio of 1.2. as 1.2 in the dots condition performed better on the number comparison task (as reflected by lower Weber fractions) than individuals whose brain did not discriminate this ratio. This observation was confirmed by correlations analyses (albeit bearing in mind the relatively small sample size of the current study for such analyses), in that participants with a greater neural sensitivity to numerical changes using controlled dots featured better behavioral ANS acuity (as in Guillaume et al., 2018). This finding supports that cognitive processes indexing spontaneous numerical discrimination recorded with EEG are related to explicit numerical 
judgements. It should, however, be noted that no significant correlation was observed between ANS acuity and the neural sensitivity to numerical discrimination when using

391 pictures (instead of controlled dots) as stimuli. Apart from null findings related to small

392 sample size, an explanation for this discrepancy could be differences in the stimuli formats.

393 Using the same stimuli in the number comparison task than in the controlled dots condition

394 might have favoured the relation between brain sensitivity and behavioural performance. If this explanation applies, using more complex and colorful stimuli instead of dots in the behavioural task should then favour a correlation with the neural sensitivities to numerical changes in the pictures condition. In any case, the current correlation outcomes should be considered with caution and verified using larger sample sizes.

It is worth mentioning that brain amplitudes tagged to the numerical fluctuation were systematically stronger in the pictures condition than in the dots condition in our dataset. In other words, pictures elicited stronger synchronisation to the numerosity changes. This is not totally surprizing because we deliberately did not control for potential confounding factors related to non-numerical dimensions in this condition (see Gebuis et al., 2016; Leibovitch et al., 2016). It is likely that visual information inherently correlated with numerosity provided additional congruent information regarding the numerical aspect of the arrays. On the contrary, it does not seem that the complexity of the pictures (such as containing subelement) impeded brain synchronization to numerosity. This is in line with existing data 408 showing that number is a salient property of the visual environment (Anobile et al., 2019;

409 Cicchini et al., 2016; Kluth \& Zetzsche, 2016).

$410 \quad$ Nonetheless, despite the aforementioned differences between controlled dots and

411 colorful pictures, similar electrophysiological response patterns were observed regardless of 412 stimulus complexity, suggesting that both conditions indexed ANS acuity and can therefore 413 be used as neural signatures of the number sense. More concretely, we observed a significant 
414 effect of ratio in both conditions. In addition, cerebral responses reflecting numerical

415 discrimination were on average significant from the ratio of 1.2 onward for both dots and

416 pictures stimuli, which also corresponds to the ratio at which averaged behavioural

417 performances were significantly above $75 \%$ correct in the number comparison task.

418 Moreover, the neural sensitivities to numerical changes were related across both conditions.

419 Namely, cerebral responses in the dots condition tended to correlate with those in the pictures

420 condition, indicating common brain processes regardless of stimulus format. Overall, these

421 findings suggest that the neural marker of numerical discrimination is quite robust to stimulus

422 format in an inter-individual perspective. This is a promising avenue for future research, as

423 appealing pictures instead of controlled dots could be used to measure ANS acuity. The

424 possibility to use appealing stimuli together with the fact that the neural marker is extracted

425 using a passive viewing task of very short duration makes the present paradigm especially

suitable for younger populations including infants, which are usually easily distracted.

A final interesting finding is that the neural sensitivities to numerical discrimination related to a measure of math fluency regardless of whether dots or appealing pictures were displayed. Since the neural marker of ANS acuity was extracted using a passive viewing task devoid of any explicit numerical judgments, these findings further hint at the importance of the number sense for mathematical development. Nonetheless, it should be noted here that although the correlations between the frequency-tagged EEG signal and math fluency were corrected for multiple comparisons as well as confirmed with group comparisons, they should

434 be considered with caution due to the relatively small sample size of the present study and replicated with more participants to validate the present outcomes. In spite of a power analysis justifying the current sample size for the extraction of a neural marker of numerical

437 discrimination using very short sequences as well as for the assessment of any potential 
438 effects of stimulus format (i.e., dots versus pictures), it was likely too small to draw any strong conclusions from the correlation analyses.

Our small sample size of $n=21$ could then also account for the lack of correlation between the behavioural performances on the non-symbolic number comparison task and math fluency, since the effect size for this relation was reported to be below .3 (see Chen $\&$

443 Li, 2014; and see Schneider et al., 2016; for meta-analyses). Albeit, such a null correlation would also be in line with many previous studies that failed to report a significant relation between ANS acuity, as assessed using non-symbolic number comparison tasks, and math ability in adults (e.g., Inglis et al., 2011; see Chen \& Li, 2014; and see Schneider et al., 2016). Considering that more general cognitive processes (e.g., executive control functions, see

448 Cragg \& Gilmore, 2014; Gilmore et al., 2013) were suggested to potentially moderate the relation between explicit measures of the number sense and math fluency, further studies should investigate the potential role of executive functions in the relationship between spontaneous visual number processing and more elaborate mathematical knowledge. It goes without saying that these studies should focus on larger sample sizes as well as different populations, including children. Finding any significant relations between the cerebral responses reflecting numerical discrimination and explicit numerical processing as well as math fluency in the latter individuals would open up the practicality of the present paradigm even further, by suggesting that it could be used to predict math ability in young children. In this regard, it should also be verified whether frequency-tagged EEG signals reflecting numerical discrimination can predict math fluency in out-of-sample participants, as suggested by Gabrieli, Ghosh, \& Whitfield-Gabrieli, (2015). 


\section{Participants and ethical considerations}

Twenty-two students from the University of Luxembourg participated in this study.

Volunteers suffering from or with a history of suffering from any neurological or

467 neuropsychological disease, any learning disability such as dyscalculia, or any uncorrected visual impairment were not allowed to participate. All participants gave written informed consent and received a remuneration of 25 euros. We excluded one participant from all analyses who did not adequately respond to the behavioural task during the EEG recording. The final sample thus consisted of twenty-one adults (eight males), with a mean age of 23.43 years $(S D=4.46$, range $=18.23-35.40)$. The study was approved by the Ethic Review Panel from the University of Luxembourg (ERP).

A statistical power analysis was conducted using the $\mathrm{G}^{*}$ Power 3 software (Faul,

475 Erdfelder, Buchner, \& Lang, 2009) to estimate the current sample size. The power analysis was conducted for a one-way repeated measures ANOVA including two levels, since we were interested in whether significant frequency-tagged EEG signals on Oz differed depending on stimulus format (i.e., dots versus pictures). The correlation between the repeated measures was set to .5 , the default value of $G^{*}$ Power, since we did not have any a priori hypotheses regarding the correlation between EEG signals recorded during the presentation of dots or pictures. Estimation was based on previously reported effect sizes in an EEG study aimed at determining a neural index of numerical sensitivity in adults (see

483 Park, 2017). In that study, large number-specific effects (i.e., $\left.\eta_{\mathrm{p}}{ }^{2}=0.186\right)$ were observed amongst others on the medial occipital electrode Oz. With an alpha $=.05$ and power $=0.95$, a sample size of $n=17$ is sufficient to detect effects at this level for comparisons between two repeated conditions. The current sample size of $n=21$ is therefore more than adequate for the

487 main objectives of this study, that is, 1) the extraction of a cerebral measure of numerical 
discrimination from posterior brain regions using very brief sequences and 2) the assessment of the dependence of this neural marker on stimulus format (i.e., dots versus pictures). Apparatus and procedure We used MATLAB (The MathWorks) with the Psychophysics Toolbox extensions (Brainard, 1997; Kleiner et al., 2007) to display the computerized tasks and record

493 behavioural data. Participants were comfortably seated at 1 meter from the screen, with their gaze directed at the centre of the screen (24', LED monitor, $100 \mathrm{Hertz}(\mathrm{Hz})$ refresh rate, $1 \mathrm{~ms}$ response time). Screen resolution was $1280 \times 1024$ px. Behavioural tasks were administered first. All participants started with the math fluency task. After that, they took part in the EEG recording session, consisting of two non-symbolic number conditions administered in counter-balanced order across the participants.

\section{Non-symbolic number comparison task}

Participants simultaneously saw two dot arrays and they were instructed to determine as accurately as possible the array containing the largest number of dots. The onset of each

502 trial was indicated by a fixation cross appearing $500 \mathrm{~ms}$ before the dots. The arrays only

503 remained on the screen for a maximal duration of $800 \mathrm{~ms}$ to prevent participants from counting the dots. We used an active mask until response to suppress retinal persistence. Inter-stimulus interval lasted $400 \mathrm{~ms}$.

We generated non-symbolic number pairs with the help of NASCO (Guillaume,

507 Schiltz, \& Van Rinsveld, 2020). Stimulus pairs were divided into four categories. In a first

508 category, total Area and convex Hull were equalized across the stimulus pair; in a second category, total area and mean occupancy were equalized; in a third category, item size and convex hull were equalized; and in the final category, item size and mean occupancy were

511 equalized. In total, 192 stimulus pairs were generated, each comprising an array of 30 dots as

512 the standard quantity to which the second array (of the pair) had to be compared. The 
513 corresponding arrays were created by computing six numerical ratios (from 1.1 to 1.6 with an

514 incremental step of 0.1 ), starting from the standard quantity of 30 dots in both increasing and

515 decreasing manners. The number of dots in the second array thus ranged from 19 to 48 . There

516 were thirty-two pairs for each of the six ratios (i.e., sixteen where the second quantity was

517 below and above 30 respectively). All dots had the same size within an array. Stimulus order

518 and the position of the correct response (either left or right) were randomized.

519 We computed individual Weber fractions $(w)$ by adjusting a Gaussian cumulative

520 probability distribution function using nonlinear regression, based on the Levenberg-

521 Marquardt-Fletcher nonlinear least square iterative method (as in Guillaume et al., 2018; see

522 Halberda et al., 2008; Pica et al., 2004; for more detailed methodological considerations). We

523 considered $w$ as a behavioural measure of explicit numerical judgments.

524

525

526

527

528

529

530

531

532

533

534

535

536

537

\section{Math fluency task}

We used the Tempo-Test Rekenen (TTR, De Vos, 1992) to evaluate math fluency.

This timed paper-and-pencil calculation test consists of five columns of forty arithmetic problems, with each column corresponding to one arithmetic operation (addition, subtraction, multiplication, division) and to a mix of all operations. The item difficulty increases throughout each column, from single-digit arithmetic facts to more complex two-digit problems. Each column was presented on a separate sheet of paper and participants were instructed to write down as many correct responses as they could within one minute.

Participants were awarded one point per correct answer. We considered the raw sum of correct responses across all five subtests $(\max =200)$ as a measure of math fluency.

\section{Fast Periodic Visual Stimulation}

\section{Material and procedure}

Participants were instructed to keep their gaze on a small fixation diamond that was continuously displayed at the centre of the screen. We used stimuli that subtended a maximal 
visual angle of nine degree. Stimulus presentation followed a sinusoidal contrast modulation from 0\% to 100\% (see Figure 1A, as in De Keyser, Mouraux, Quek, Torta, \& Legrain, 2018; or in Lochy, de Heering, \& Rossion, 2017). The base frequency rate was $10 \mathrm{~Hz}$, corresponding to the display of 10 stimuli per second. Every stimulation sequence lasted 64 seconds, including 60 seconds of recording and 2 seconds of fade-in and fade-out respectively, which were not analysed.

During each stimulation sequence, we alternated between two numerical quantities.

Alternation thus occurred at a frequency rate of $5 \mathrm{~Hz}$. One of the numerical quantities used during alternation (i.e., the carrier) was kept constant at a numerosity of 10 items. The second numerosity changed every ten seconds, thus in total 6 times during each stimulation sequence of 60 seconds. During the first 10 seconds, the interspersed numerical quantity was identical to the fixed numerosity. It then linearly increased from a ratio of 1.1 (i.e., eleven items) to a ratio of 1.5 (i.e., fifteen items) with an incremental step of 0.1 . This increasing fluctuation within a single stimulation sequence is based on the sweep visual evoked potential technique (Almoqbel, Leat, \& Irving, 2008; Regan, 1977; see also, Hemptinne, Liu-Shuang, Yuksel, \& Rossion, 2018; Liu-Shuang, Ales, Rossion, \& Norcia, 2015; Ridder, Waite, \& Melton, 2014). We created two conditions. In the first condition, we presented randomly arranged dots, created with NASCO (Guillaume et al., 2020). We statistically verified that random fluctuations related to the non-numerical visual dimensions were not periodic within our stimulation sequences of 60 seconds by computing the Fast-Fourier Transformation (FFT) of the values taken by all dimensions over the time (see the supplementary material from Van

559 Rinsveld et al., 2020). For each condition, we selected one stimuli sequence in which the averaged periodicity value of the numerical dimension was significant whereas the averaged

561 periodicity values of all other non-numerical dimensions were non-significant. In this setting, 562 visual features were thus drastically controlled. 
564 pictures bought on the Fotolia database. These pictures consisted of colourful food items,

565 such as fruits and vegetables (see Figure 1). Pictures were randomly drawn (without

566 replacement) to form an array with the desired number of food items. To increase variability,

567 we let the size of each item stochastically vary (25\% of variation from its base size, which

568 was heterogeneous as a function of the item) and we also added some random mirroring

569 effect. Importantly, we deliberately choose food items that might contain sub-elements such

570 as green beans (containing three pods) or pizza slices (containing three pepperonis).

571 Therefore, and conversely to our first condition, this second condition was not designed to

572 control for visual cues but rather illustrate stimulus complexity.

573 Both conditions were repeated ten times in every participant, entailing twenty

574 stimulation sequences (i.e., 20 minutes of recording) per participants.

575 To verify that participants kept their gaze at the centre the screen, the small fixation

576 diamond randomly changed colour from blue to red. Colour changes occurred from six to

577 eight times during a stimulation sequence. Participants were instructed to press a button with

578 their right forefinger. Based on these presses, we were able to verify participants' compliance 579 with the task instruction.

\section{$580 \quad$ EEG acquisition}

The setup was similar to the one used in Guillaume et al. (2018). We acquired EEG

582 data using a 64-channel BioSemi ActiveTwo system at $2048 \mathrm{~Hz}$ (BioSemi B. V., Amsterdam,

583 The Netherlands). We positioned the electrodes on the cap according to the standard 10-20

584 system locations (for position coordinates, see http://www.biosemi.com). We used two

585 additional electrodes, the Common Mode Sense (CMS) active electrode and the Driven Right

586 Leg (DRL) passive electrode, as reference and ground electrodes, respectively. We hold

587 offsets of the electrodes below $40 \mathrm{mV}$ during acquisition. 
Analyses were conducted with Letswave 6 (http://nocions.webnode.com/letswave).

590 Data files were down-sampled from $2048 \mathrm{~Hz}$ to $512 \mathrm{~Hz}$ for faster processing. We then

591 filtered the data with a 4-order band-pass Butterworth filter (0.1 to $100 \mathrm{~Hz})$. In three

592 participants, one channel had to be interpolated across all conditions using the three closest

593 neighbouring electrodes due to excessive noise in the signal throughout the entire EEG

594 recording. We did not correct the EEG signal for the presence of ocular artefacts. The data was then re-referenced to the common average.

We segmented the signal into six chunks of 10 seconds, corresponding to the six

597 different numerical quantities displayed in alternation with the fixed numerosity of 10 items.

598 The EEG signal from each of the six different ratios with respect to the fixed quantity was then averaged across the ten repetitions for each of the two conditions per participant. A Fast

600 Fourier Transform (FFT) was applied to the signal to extract amplitude spectra for the 64 601 channels with a frequency resolution of $0.1 \mathrm{~Hz}$.

602 Based on these frequency spectra, we computed three measures to determine whether 603 and how the brain specifically responded to the alternation of numerical quantities at $5 \mathrm{~Hz}$ 604 during each of the two conditions. First, we computed Baseline-Corrected Amplitudes (BCA) by subtracting from the $5 \mathrm{~Hz}$ bin the mean amplitude of its twenty surrounding bins (ten on each side, excluding the immediately adjacent bins, and the two extreme values). BCA are

607 thus expressed in microvolt and can therefore be considered to quantify changes within the 608 EEG signal (Dzhelyova \& Rossion, 2014a, 2014b; Guillaume et al., 2018; Liu-Shuang et al., 2014; Retter \& Rossion, 2016). BCA were used to depict the scalp topographies in Figure 2.

611 frequency bin with the average amplitude of its respective twenty surrounding bins 
612 (excluding the immediately adjacent bins, and the two extreme values). We used SNRs to

613 illustrate the spectra in Figure 2.

614 Lastly, we computed a Z-score to quantify the statistical significance of the brain

615 response to the numerosity change at $5 \mathrm{~Hz}$. More concretely, we applied a Z-transformation

616 to the $5 \mathrm{~Hz}$ bin as a function of its surrounding twenty bins representing the noise level. This

617 computation yielded a Z-score of the brain response specific to the experimental

618 manipulation at $5 \mathrm{~Hz}$, which can be interpreted as the neural response to the quantity change

619 (i.e., numerical discrimination). A Z-score larger than the threshold of $1.64(p<.05$, one-

620 tailed, testing signal level > noise level) indicates a significant response to the experimental

621 manipulation. The Z-scores were used to conduct all statistical analyses.

622

Ethical statement

623

We followed APA ethical standards to conduct the present study. The Ethic Review

624 Panel from the University of Luxembourg (ERP) approved the methodology and the

625 implementation of the experiment before the start of data collection.

626

Acknowledgement

627

We would like to thank Amna Skrozic for her invaluable help with data collection.

628

This is a preprint of an article published in Scientific Reports. The final authenticated version is now available online.

630

Funding

631

This study was funded by FHSE research-budget allocated to the CNS lab.

632

\section{Author contributions}

633

M.G. and C.S. developed the study concept and design. M.G. wrote the EEG script.

634 C.G. collected data and performed statistical analysis. C.G. and M.G. wrote the first draft of 635 the manuscript. All authors discussed the results and revised the manuscript.

636

\section{Data availability}




\section{References}

639 1. Dehaene, S. (2001). Précis of the number sense. Mind and Language, 16(1), 16-36.

640 2. Feigenson, L., Dehaene, S., \& Spelke, E. (2004). Core systems of number. Trends in 641 Cognitive Sciences, 8(7), 307-314. https://doi.org/10.1016/j.tics.2004.05.002

642 3. Gallistel, C. R., \& Gelman, R. (2000). Non-verbal numerical cognition : From reals to 643 integers. Trends in Cognitive Sciences, 4(2), 59-65.

644 4. Dehaene, S. (2003). The neural basis of the Weber-Fechner law : A logarithmic mental 645 number line. Trends in Cognitive Sciences, 7(4), 145-147. https://doi.org/10.1016/S1364$646 \quad 6613(03) 00055-X$

647 5. Cantlon, J. F., Cordes, S., Libertus, M. E., \& Brannon, E. M. (2009). Comment on "Log 648 or linear? Distinct intuitions of the number scale in Western and Amazonian indigene 649 cultures". Science, 323(5910), 38b-38b.

650 6. Piantadosi, S. T. (2016). A rational analysis of the approximate number system.

651 Psychonomic Bulletin \& Review, 23(3), 877-886. https://doi.org/10.3758/s13423-015$652 \quad 0963-8$

653 7. Halberda, J., Mazzocco, M. M. M., \& Feigenson, L. (2008). Individual differences in 654 non-verbal number acuity correlate with maths achievement. Nature, 455(7213), 665-668. 655 https://doi.org/10.1038/nature07246

656 8. Inglis, M., Attridge, N., Batchelor, S., \& Gilmore, C. (2011). Non-verbal number acuity 657 correlates with symbolic mathematics achievement: But only in children. Psychonomic 658 Bulletin \& Review, 18(6), 1222-1229. https://doi.org/10.3758/s13423-011-0154-1

659 9. Mussolin, C., Mejias, S., \& Noël, M.-P. (2010). Symbolic and nonsymbolic number 660 comparison in children with and without dyscalculia. Cognition, 115(1), 10-25. 661 https://doi.org/10.1016/j.cognition.2009.10.006 
662 10. DeWind, N. K., \& Brannon, E. M. (2012). Malleability of the approximate number 663 system : Effects of feedback and training. Frontiers in Human Neuroscience, 6. 664 https://doi.org/10.3389/fnhum.2012.00068

665 11. Sasanguie, D., Göbel, S. M., Moll, K., Smets, K., \& Reynvoet, B. (2013). Approximate 666 number sense, symbolic number processing, or number-space mappings : What underlies 667 mathematics achievement? Journal of Experimental Child Psychology, 114(3), 418-431. $668 \quad$ https://doi.org/10.1016/j.jecp.2012.10.012

669 12. Price, G. R., Palmer, D., Battista, C., \& Ansari, D. (2012). Nonsymbolic numerical 670 magnitude comparison : Reliability and validity of different task variants and outcome 671 measures, and their relationship to arithmetic achievement in adults. Acta Psychologica, 672 140(1), 50-57. https://doi.org/10.1016/j.actpsy.2012.02.008

13. Chen, Q., \& Li, J. (2014). Association between individual differences in non-symbolic 674 number acuity and math performance : A meta-analysis. Acta Psychologica, 148, 163-172. https://doi.org/10.1016/j.actpsy.2014.01.016

14. Schneider, M., Beeres, K., Coban, L., Merz, S., Susan Schmidt, S., Stricker, J., \& De Smedt, B. (2016). Associations of non-symbolic and symbolic numerical magnitude processing with mathematical competence : A meta-analysis. Developmental Science, n/a-n/a. https://doi.org/10.1111/desc.12372

15. DeWind, N. K., \& Brannon, E. M. (2016). Significant Inter-Test Reliability across Approximate Number System Assessments. Frontiers in Psychology, 7. https://doi.org/10.3389/fpsyg.2016.00310

16. Norris, J. E., \& Castronovo, J. (2016). Dot Display Affects Approximate Number System 684 Acuity and Relationships with Mathematical Achievement and Inhibitory Control. PLOS ONE, 11(5), e0155543. https://doi.org/10.1371/journal.pone.0155543 
686

687

688

689

690

691

692

693

694

695

696

697

698

699

700

701

702

703

704

705

706

707

708

17. Szücs, D., Nobes, A., Devine, A., Gabriel, F. C., \& Gebuis, T. (2013). Visual stimulus parameters seriously compromise the measurement of approximate number system acuity and comparative effects between adults and children. Frontiers in Psychology, 4. https://doi.org/10.3389/fpsyg.2013.00444

18. Roquet, A., \& Lemaire, P. (2019). Strategy variability in numerosity comparison task : A study in young and older adults. Open Psychology, 1(1), 152-167. https://doi.org/10.1515/psych-2018-0011

19. Gebuis, T., Cohen Kadosh, R., \& Gevers, W. (2016). Sensory-integration system rather than approximate number system underlies numerosity processing : A critical review. Acta Psychologica, 171, 17-35. https://doi.org/10.1016/j.actpsy.2016.09.003

20. Cragg, L., \& Gilmore, C. (2014). Skills underlying mathematics : The role of executive function in the development of mathematics proficiency. Trends in Neuroscience and Education, 3(2), 63-68. https://doi.org/10.1016/j.tine.2013.12.001

21. Gilmore, C., Attridge, N., Clayton, S., Cragg, L., Johnson, S., Marlow, N., Simms, V., \& Inglis, M. (2013). Individual Differences in Inhibitory Control, Not Non-Verbal Number Acuity, Correlate with Mathematics Achievement. PLoS ONE, 8(6), e67374.

22. Smets, K., Gebuis, T., Defever, E., \& Reynvoet, B. (2014). Concurrent validity of approximate number sense tasks in adults and children. Acta Psychologica, 150, 120-128. https://doi.org/10.1016/j.actpsy.2014.05.001

23. Anobile, G., Guerrini, G., Burr, D. C., Monti, M., Del Lucchese, B., \& Cicchini, G. M. (2019). Spontaneous perception of numerosity in pre-school children. Proceedings of the Royal Society B: Biological Sciences, 286(1906), 20191245.

https://doi.org/10.1098/rspb.2019.1245 
24. Cicchini, G. M., Anobile, G., \& Burr, D. C. (2016). Spontaneous perception of numerosity in humans. Nature Communications, 7, 12536. https://doi.org/10.1038/ncomms12536

25. Kluth, T., \& Zetzsche, C. (2016). Numerosity as a topological invariant. Journal of vision, 16(3), 30-30.

26. Stoianov, I., \& Zorzi, M. (2012). Emergence of a « visual number sense » in hierarchical generative models. Nature Neuroscience, 15(2), 194-196. https://doi.org/10.1038/nn.2996

27. Burr, D., \& Ross, J. (2008). A Visual Sense of Number. Current Biology, 18(6), 425-428. https://doi.org/10.1016/j.cub.2008.02.052

28. Ferrigno, S., Jara-Ettinger, J., Piantadosi, S. T., \& Cantlon, J. F. (2017). Universal and uniquely human factors in spontaneous number perception. Nature Communications, 8 , 13968. https://doi.org/10.1038/ncomms13968

29. Anobile, G., Tomaiuolo, F., Campana, S., \& Cicchini, G. M. (2020). Three-systems for visual numerosity: A single case study. Neuropsychologia, 136, 107259. https://doi.org/10.1016/j.neuropsychologia.2019.107259

30. Rossion, B. (2014). Understanding individual face discrimination by means of fast periodic visual stimulation. Experimental Brain Research, 232(6), 1599-1621. https://doi.org/10.1007/s00221-014-3934-9

31. Adrian, E. D., \& Matthews, B. H. (1934). The Berger rhythm: Potential changes from the occipital lobes in man. Brain, 57(4), 355-385.

32. Regan, D. (1977). Steady-state evoked potentials. Journal of the Optical Society of America, 67(11), 1475-1489.

33. Norcia, A. M., Appelbaum, L. G., Ales, J. M., Cottereau, B. R., \& Rossion, B. (2015). The steady-state visual evoked potential in vision research: A review. Journal of Vision, 15(6), 4-4. https://doi.org/10.1167/15.6.4 
34. Regan, D. (1989). Human brain electrophysiology: Evoked potentials and evoked magnetic fields in science and medicine. Amsterdam, the Netherlands: Elsevier.

35. Liu-Shuang, J., Norcia, A. M., \& Rossion, B. (2014). An objective index of individual face discrimination in the right occipito-temporal cortex by means of fast periodic oddball stimulation. Neuropsychologia, 52, 57-72. https://doi.org/10.1016/j.neuropsychologia.2013.10.022

36. Retter, T. L., \& Rossion, B. (2016). Visual adaptation provides objective electrophysiological evidence of facial identity discrimination. Cortex, 80, 35-50. https://doi.org/10.1016/j.cortex.2015.11.025

37. Dzhelyova, M., Jacques, C., \& Rossion, B. (2016). At a Single Glance : Fast Periodic Visual Stimulation Uncovers the Spatio-Temporal Dynamics of Brief Facial Expression Changes in the Human Brain. Cerebral Cortex. https://doi.org/10.1093/cercor/bhw223

38. Lochy, A., Van Belle, G., \& Rossion, B. (2015). A robust index of lexical representation in the left occipito-temporal cortex as evidenced by EEG responses to fast periodic visual stimulation. Neuropsychologia, 66, 18-31. https://doi.org/10.1016/j.neuropsychologia.2014.11.007

39. Lochy, A., Jacques, C., Maillard, L., Colnat-Coulbois, S., Rossion, B., \& Jonas, J. (2018). Selective visual representation of letters and words in the left ventral occipito-temporal cortex with intracerebral recordings. Proceedings of the National Academy of Sciences, 115(32), E7595-E7604. https://doi.org/10.1073/pnas.1718987115

40. De Keyser, R., Mouraux, A., Quek, G. L., Torta, D. M., \& Legrain, V. (2018). Fast periodic visual stimulation to study tool-selective processing in the human brain. Experimental Brain Research, 236(10), 2751-2763. https://doi.org/10.1007/s00221-018$5331-2$ 
41. Guillaume, M., Poncin, A., Schiltz, C., \& Van Rinsveld, A., (submitted). Spontaneous and automatic processing of magnitude and parity information of Arabic digits: A frequency-tagging EEG study. Preprint available at https://www.biorxiv.org/content/10.1101/2019.12.26.888651v2

42. Guillaume, M., Mejias, S., Rossion, B., Dzhelyova, M., \& Schiltz, C. (2018). A rapid, objective and implicit measure of visual quantity discrimination. Neuropsychologia, 111, 180-189.

43. Van Rinsveld, A., Guillaume, M., Kohler, P. J., Schiltz, C., Gevers, W., \& Content, A. (2020). The neural signature of numerosity by separating numerical and continuous magnitude extraction in visual cortex with frequency-tagged EEG. Proceedings of the National Academy of Sciences, 117(11), 57265732. https://doi.org/10.1073/pnas.1917849117

44. Park, J. (2017). A neural basis for the visual sense of number and its development: A steady-state visual evoked potential study in children and adults. Developmental Cognitive Neuroscience.

45. Lucero, C., Brookshire, G., Sava-Segal, C., Bottini, R., Goldin-Meadow, S., Vogel, E. K., \& Casasanto, D. (2020). Unconscious Number Discrimination in the Human Visual System. Cerebral Cortex. https://doi.org/10.1093/cercor/bhaa155

46. Cook, R. Dennis (February 1977). "Detection of Influential Observations in Linear Regression". Technometrics. American Statistical Association. 19 (1), 15-18.

47. Leibovich, T., Katzin, N., Harel, M., \& Henik, A. (2016). From 'sense of number' to 'sense of magnitude' - The role of continuous magnitudes in numerical cognition. Behavioral and Brain Sciences, 1-62. 
781

782

783

784

785

786

787

788

789

790

791

792

793

794

795

796

797

798

799

800

801

802

803

804

48. Gabrieli, J. D., Ghosh, S. S., \& Whitfield-Gabrieli, S. (2015). Prediction as a humanitarian and pragmatic contribution from human cognitive neuroscience. Neuron, 85(1), 11-26.

49. Faul, F., Erdfelder, E., Buchner, A., \& Lang, A. G. (2009). Statistical power analyses using G*Power 3.1: Tests for correlation and regression analyses. Behavior Research Methods, 41(4), 1149-1160.

50. Brainard, D. H. (1997). The psychophysics toolbox. Spatial Vision, 10(4), 433-436.

51. Kleiner, M., Brainard, D., Pelli, D., Ingling, A., Murray, R., \& Broussard, C. (2007). What's new in Psychtoolbox-3? Perception, 36(14), 1-16.

52. Guillaume, M., Schiltz, C., \& Van Rinsveld, A. (2020). NASCO: A new method and program to generate dot arrays for non-symbolic number comparison tasks. Journal of Numerical Cognition.

53. Pica, P., Lemer, C., Izard, V., Dehaene, S., 2004. Exact and approximate arithmetic in an amazonian indigene group. Science 306 (5695), 499-503.

54. De Vos, T. (1992). Test voor het vaststellen van het rekenvaardigheidsniveau der elementaire bewerkingen (automatisering) voor het basis en voortgezet onderwijs: Handleiding [Test to determine the mathematics ability level for elementary operations (automatization) in primary and secondary education: Manual]. Nijmegen, the Netherlands: Berkhout.

55. Lochy, A., de Heering, A., \& Rossion, B. (2017). The non-linear development of the right hemispheric specialization for human face perception. Neuropsychologia, 10.1016/j.neuropsychologia.2017.06.029

56. Almoqbel, F., Leat, S. J., \& Irving, E. (2008). The technique, validity and clinical use of the sweep VEP. Ophthalmic and Physiological Optics, 28(5), 393-403. 
57. Hemptinne, C., Liu-Shuang, J., Yuksel, D., \& Rossion, B. (2018). Rapid Objective Assessment of Contrast Sensitivity and Visual Acuity With Sweep Visual Evoked Potentials and an Extended Electrode Array. Investigative Opthalmology \& Visual Science, 59(2), 1144.

58. Liu-Shuang, J., Ales, J., Rossion, B., \& Norcia, A. M. (2015). Separable effects of inversion and contrast-reversal on face detection thresholds and response functions: A sweep VEP study. Journal of Vision, 15(2), 11-11.

59. Ridder, W. H., Waite, B. S., \& Melton, T. F. (2014). Comparing enfant and PowerDiva sweep visual evoked potential (sVEP) acuity estimates. Documenta Ophthalmologica, 129(2), 105-114.

60. Dzhelyova, M., \& Rossion, B. (2014a). Supra-additive contribution of shape and surface information to individual face discrimination as revealed by fast periodic visual stimulation. Journal of Vision, 14, 15.

61. Dzhelyova, M., \& Rossion, B. (2014b). The effect of parametric stimulus size variation on individual face discrimination indexed by fast periodic visual stimuliation. $B M C$ Neuroscience, 15, 87.

62. Guillaume, M., \& Van Rinsveld, A. (2018). Comparing Numerical Comparison Tasks: A Meta-Analysis of the Variability of the Weber Fraction Relative to the Generation Algorithm. Frontiers in Psychology, 9, doi.org/10.3389/fpsyg.2018.01694. 


\title{
A robust electrophysiological marker of spontaneous numerical
}

\author{
discrimination
}

Carrie Georges $^{\mathrm{a}^{*}}$, Mathieu Guillaume ${ }^{\mathrm{b}}, \&$ Christine Schiltz ${ }^{\mathrm{a}}$

\section{Supplementary Material}

\section{FPVS recording}

\section{Instruction compliance for each condition}

Repeated measures ANOVAs indicated a main effect of condition (dots vs. pictures) on both detection rate $\left(\mathrm{F}(1,20)=21.99, \mathrm{p}<.001, \eta_{\mathrm{p}}{ }^{2}=.52\right)$ and $\mathrm{RT}(\mathrm{F}(1,20)=49.91, \mathrm{p}<$ $\left..001, \eta_{\mathrm{p}}{ }^{2}=.71\right)$. Participants missed fewer color changes in the dots condition (dots: misses $=$ $0.62 \%, \mathrm{SD}=1.0 \%$ vs. pictures: misses $=7.85 \%, \mathrm{SD}=6.87 \%)$ and they were also faster to detect these changes when dots were displayed (dots: $\mathrm{RT}=467 \mathrm{~ms}, \mathrm{SD}=44 \mathrm{~ms}$ vs. pictures: $\mathrm{RT}=552 \mathrm{~ms}, \mathrm{SD}=67 \mathrm{~ms})$.

\section{Response on medial occipital and other posterior electrodes}

To compare frequency-tagged EEG responses in the medial occipital cortex to the remaining posterior areas, we pooled the EEG signal from all the posterior electrodes into four regions of interest (ROIs): the medial occipital (MO: O1, O2, Oz, Iz), medial occipitoparietal (MOP: Pz, POz, PO3, PO4), left occipito-parietal (LOP: P5, P7, P9, PO7) and right occipito-parietal (ROP: P6, P8, P10, PO8) regions (Liu-Shuang, Norcia, \& Rossion, 2014). Supplementary Table 1 depicts the Z-score values averaged across all participants for each ratio and condition in these four posterior ROIs. The strongest cerebral responses at $5 \mathrm{~Hz}$ across all numerical ratios were on average observed over medial occipital electrodes in both the dots and pictures conditions. Similar frequency-tagged cerebral response patterns, in terms of Baseline-Corrected Amplitudes (BCA, see Methods), across the different ratios were observed on each of the medial occipital electrodes (i.e., Iz, O1, O2, and $\mathrm{Oz}$ ) in both the dots and pictures conditions (see Supplementary Figure 1). 


\begin{tabular}{|c|c|c|c|c|c|c|c|c|c|c|c|c|c|c|}
\hline \multirow[b]{2}{*}{ Electrode } & \multicolumn{7}{|c|}{ Dots } & \multicolumn{7}{|c|}{ Pictures } \\
\hline & 1.0 & 1.1 & 1.2 & 1.3 & 1.4 & 1.5 & All & 1.0 & 1.1 & 1.2 & 1.3 & 1.4 & 1.5 & All \\
\hline LOP & 0.23 & 0.60 & 0.36 & 0.23 & 0.25 & 1.21 & 0.48 & 0.59 & 1.24 & 1.62 & 1.08 & 2.41 & 2.44 & 1.56 \\
\hline MO & -0.37 & 0.60 & 1.46 & 1.94 & 1.52 & 3.96 & 1.52 & 0.04 & 1.33 & 2.87 & 3.75 & 3.54 & 4.07 & 2.60 \\
\hline DD & -0.22 & 0.15 & 0.41 & 0.96 & 1.67 & 2.58 & 0.92 & 0.29 & 0.87 & 2.64 & 2.65 & 2.35 & 1.80 & 1.77 \\
\hline ROP & -0.31 & 0.53 & 0.31 & 0.90 & 1.08 & 3.33 & 0.97 & 0.15 & 1.76 & 2.00 & 1.66 & 2.49 & 2.89 & 1.82 \\
\hline
\end{tabular}

Supplementary Table 1. Averaged cerebral amplitudes (in Z-score) for every ratio in each condition as a

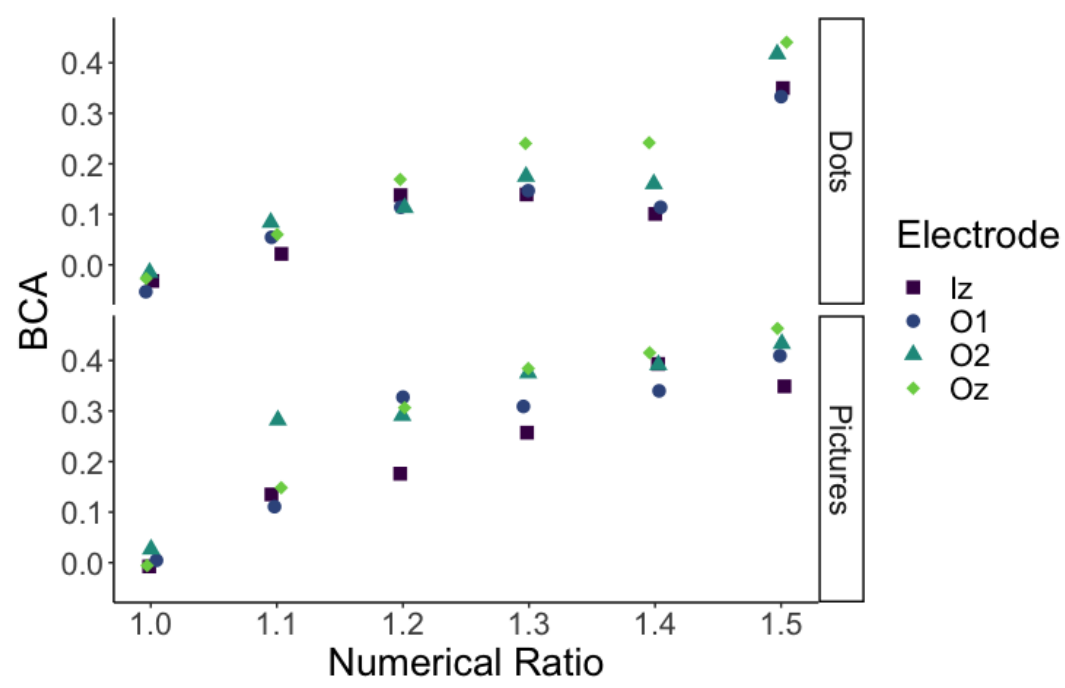

Supplementary Figure 1. Averaged cerebral amplitudes (in terms of Baseline-Corrected-Amplitudes, BCA, in $\mu \mathrm{V}$ ) on the medial occipital electrodes $\mathrm{Iz}, \mathrm{O} 1, \mathrm{O} 2$, and $\mathrm{Oz}$ as a function of numerical ratio in the Dots and

36 Pictures conditions.

\section{Relationship between the FPVS response and the behavioural measures}

To further assess the relations between the EEG signal reflecting numerical

discrimination and the behavioural measures, we additionally performed correlation analyses

40 with frequency-tagged cerebral responses at the numerical ratio 1.2 on each individual's best responding medial occipital electrode at that ratio. All correlations remained similar to when 
Oz was considered across the entire sample. Namely, in both the dots and pictures conditions, stronger cerebral responses at $5 \mathrm{~Hz}$ on each individual's best responding medial occipital electrode at 1.2 were associated with better math fluency (dots: $r=.41, p=.06$; pictures: $r=$ $.46, p=.04)$. Conversely, only in the dots condition, a relation was observed between higher EEG signals at $5 \mathrm{~Hz}$ on every individual's best responding medial occipital electrode at the ratio 1.2 and lower Weber fractions (dots: $r=-.54, p=.01$; pictures: $r=-.33, p=.15$ ). In a previous study, we also computed an index of the cerebral response across all numerical ratios and we observed that this index correlated with ANS acuity (Guillaume et al., 2018). To replicate this finding, we computed this index also in the current study. More concretely, we fitted a linear regression for both conditions, for each individual, predicting the $\mathrm{Oz}$ amplitudes at $5 \mathrm{~Hz}$ across the numerical ratios. We then extracted the coefficients relative to the numerical ratio (i.e., the slope) for every participant, with steeper slopes reflecting greater neural sensitivity to numerical discrimination. We excluded three individuals in total with negative regression slopes in either the dots or pictures condition. This exclusion criterium was based on a previous study by Guillaume et al. (2018; see Figure 4) as well as on the current group-level findings (see Figure 3), indicating that on average the magnitudes of the frequency-tagged medial occipital responses linearly increased with the magnitudes of the deviant numerosity. Such a linear increase between the EEG signals and numerical ratios is expected as it directly reflects behavioural performances on explicit 61 number comparison tasks and thereby the architecture of the ANS. It thus substantiates the claim that the present EEG signals can be considered as a reliable neural index of ANS acuity. Consequently, relations between frequency-tagged cerebral responses and numerical ratios that are not positively (but negatively) increasing might reflect a failure to adequately capture numerical discrimination capacity using the current EEG paradigm. Individuals with negative regression slopes were therefore excluded from the present correlation analyses. In 
67 accordance with previous findings (Guillaume et al., 2018) and consistent with the outcomes

68 based on the EEG amplitudes recorded for the 1.2 ratio, higher scores on the math fluency

69 task were associated with significantly more positive linear regression slopes in both the dots

$70(r=.56, p=.017)$ and the pictures $(r=.50, p=.034)$ conditions. Moreover, lower Weber

71 fractions were related to significantly steeper positive linear regression slopes in the dots $(r=$

$72-.53, p=.024)$, but not the pictures $(r=.11, p=.67)$ conditions. The linear regression slopes

73 correlated across the two conditions $(r=.52, p=.027)$, suggesting that neural responses were

74 similarly sensitive to the numerical ratio across stimuli formats. 